\title{
Molecular outflow launched beyond the disk edge
}

\author{
F. O. Alves ${ }^{1}$, J. M. Girart ${ }^{2}$, P. Caselli ${ }^{1}$, G. A. P. Franco ${ }^{3}$, B. Zhao ${ }^{1}$, W. H. T. Vlemmings ${ }^{4}$, M. G. Evans ${ }^{5}$, and L. Ricci ${ }^{6}$ \\ 1 Max-Planck-Institut für extraterrestrische Physik, Giessenbachstr. 1, 85748 Garching, Germany \\ e-mail: falves@mpe.mpg.de \\ 2 Institut de Ciències de l'Espai (CSIC-IEEC), Campus UAB, Carrer de Can Magrans S/N, 08193 Cerdanyola del Vallès, Catalonia, \\ Spain \\ 3 Departamento de Física-ICEx-UFMG, Caixa Postal 702, 30.123-970 Belo Horizonte, Brazil \\ 4 Department of Earth and Space Sciences, Chalmers University of Technology, Onsala Space Observatory, 43992 Onsala, Sweden \\ 5 School of Physics \& Astronomy, University of Leeds, LS2 9LN Leeds, UK \\ 6 Department of Physics and Astronomy, Rice University, Main Street, 77005 Houston, USA
}

Received 1 May 2017 / Accepted 5 July 2017

\begin{abstract}
One of the long-standing problems of star formation is the excess of angular momentum of the parent molecular cloud. In the classical picture, a fraction of angular momentum of the circumstellar material is removed by the magneto-centrifugally driven disk wind that is launched from a wide region throughout the disk. In this work, we investigate the kinematics in the envelope-disk transition zone of the Class I object BHB07-11, in the B59 core. For this purpose, we used the Atacama Large Millimeter/submillimeter Array in extended configuration to observe the thermal dust continuum emission $\left(\lambda_{0} \sim 1.3 \mathrm{~mm}\right)$ and molecular lines $\left(\mathrm{CO}, \mathrm{C}^{18} \mathrm{O}\right.$ and $\left.\mathrm{H}_{2} \mathrm{CO}\right)$, which are suitable tracers of disk, envelope, and outflow dynamics at a spatial resolution of $\sim 30 \mathrm{AU}$. We report a bipolar outflow that was launched at symmetric positions with respect to the disk ( $\sim 80 \mathrm{AU}$ in radius), but was concentrated at a distance of $90-130 \mathrm{AU}$ from the disk center. The two outflow lobes had a conical shape and the gas inside was accelerating. The large offset of the launching position coincided with the landing site of the infall materials from the extended spiral structure (seen in dust) onto the disk. This indicates that bipolar outflows are efficiently launched within a narrow region outside the disk edge. We also identify a sharp transition in the gas kinematics across the tip of the spiral structure, which pinpoints the location of the so-called centrifugal barrier.
\end{abstract}

Key words. stars: formation - stars: kinematics and dynamics - stars: winds, outflows - accretion, accretion disks ISM: magnetic fields

\section{Introduction}

The star formation process starts when the self-gravity of a dense molecular core dominates all the different pressure terms. The initial angular momentum in the core causes the formation of protoplanetary disks (Bodenheimer 1995; Armitage 2011). However, a significant fraction of the initial angular momentum must be removed to allow the formation of stars, mainly through two outflow-launching mechanisms: (1) the X-wind, also called stellar wind, which is launched at a few stellar radii from the star (Shu et al. 2000), and (2) the disk wind, which is launched from a wide region throughout the disk (Konigl \& Pudritz 2000). The former is likely to be responsible for the collimated high-velocity jets (Zinnecker et al. 1998), and the latter for the wide-angle outflows (Bjerkeli et al. 2016). However, the exact launching position of the disk wind is not well-constrained. Bjerkeli et al. (2016) showed that the outflow-launching locations are within the inner $\sim 25$ AU radius of the 60-100 AU disk.

The star-forming cloud B59 is located at a distance of 145 pc (Alves \& Franco 2007) and comprises the densest parts of the Pipe nebula, a molecular cloud that has been the subject of numerous investigations (e.g., Lombardi et al. 2006; Alves et al. 2007, 2008; Román-Zúñiga et al. 2007; Franco et al. 2010; Frau et al. 2010, 2012). B59 harbors a protocluster of low-mass young stellar objects (YSOs) at distinct evolutionary stages (Onishi et al. 1999; Brooke et al. 2007; Forbrich et al. 2009; Covey et al. 2010). Of these, BHB07-11 is the youngest member, a Class I object embedded in a dusty envelope with thermal emission peaking at mid-infrared bands $(70 \mu \mathrm{m})$, and its luminosity is $2.7 L_{\odot}$ (Brooke et al. 2007; Riaz et al. 2009; Forbrich et al. 2009). The large-scale kinematics ( 4000 AU) are dominated by a large outflow of molecular gas perpendicular to the rotation plane of the envelope (Duarte-Cabral et al. 2012; Hara et al. 2013).

\section{Observations}

The observations were carried out with the Atacama Large Millimeter/submillimeter Array (ALMA) using 44 antennas in an extended configuration (baselines between 0.02 and $1.6 \mathrm{~km}$ ), at an atmospheric spectral window of $230 \mathrm{GHz}(\lambda \sim 1.3 \mathrm{~mm})$. The absolute flux calibration was obtained using Titan. The bandpass calibration of the receiver response was performed by observing the quasar J1733-1304, while the relative phase and amplitude gain solutions were obtained from the quasar J1700-2610. Owing to the high variability of the calibrators, the uncertainty on the absolute flux density scale is about $7 \%$.

The observing setup contained four spectral windows, one of which was dedicated to continuum observations (in timedomain mode, TDM), and the other three dedicated to molecular line observations (in frequency-domain mode, FDM). The total bandwidth in continuum mode, taking into account the TDM window plus the line-free channels of the FDM windows, was about $2.3 \mathrm{GHz}$, leading to an rms of $62 \mu \mathrm{Jy} \mathrm{beam}^{-1}$. The 


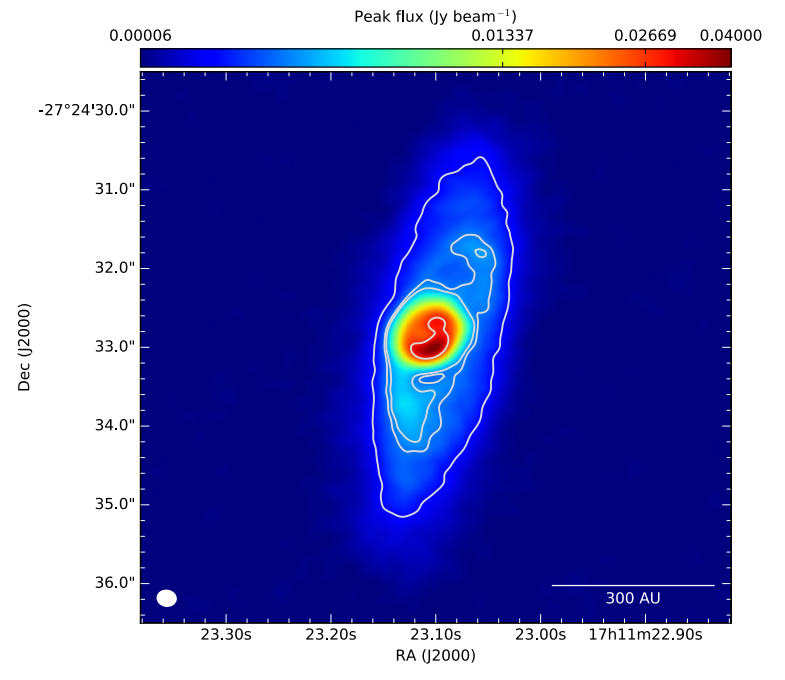

Fig. 1. Dust continuum emission with contours of $30,60,70$, and 450 times the noise level $\sigma\left(1 \sigma=62 \mu \mathrm{Jy}_{\text {beam }}{ }^{-1}\right)$ The intensity contrast between the envelope (and spirals) and the disk is clear.

molecular lines were chosen in order to probe gas at distinct states of temperature and density. We observed carbon monoxide (CO $J=2-1$ ) because of its high abundance with respect to other molecules, $\mathrm{C}^{18} \mathrm{O}(J=2-1)$ and $\mathrm{H}_{2} \mathrm{CO}\left(J=3_{0,3}-2_{0,2}\right)$, which are tracers of dense gas $\left(n \sim 10^{4-6} \mathrm{H}_{2}\right.$ particles per $\mathrm{cm}^{3}$ ). The spectral resolution is $0.32 \mathrm{~km} \mathrm{~s}^{-1}$ for the $\mathrm{CO}$ data and $0.17 \mathrm{~km} \mathrm{~s}^{-1}$ for the $\mathrm{H}_{2} \mathrm{CO}$ data, with an rms noise for the linefree channels of 0.4 and $1.5 \mathrm{mJy}^{\text {beam }}{ }^{-1}$, respectively.

The data were calibrated and imaged using CASA ${ }^{1}$. The final continuum maps were self-calibrated and improved by about $40 \%$ in noise level. Our observations provide a detailed view of the disk-envelope structure and inner kinematics for BHB07-11. The processed maps have a spatial resolution of $\sim 32 \mathrm{AU}$.

\section{Results}

\subsection{Dust continuum emission}

The dust continuum emission reveals two distinct components exhibiting significant irregularities (Fig. 1). First, there is a bright and sharp compact disk with a radius of $\sim 80 \mathrm{AU}$. This value is within the typical range of radii found in YSO disks (e.g., Cotten \& Song 2016). The disk is slightly elongated along a position angle (PA) of $138^{\circ}$ (east of north), and it encompasses a strong azimuthal asymmetry: the brightest emission is not at the disk center, but is located to the southwest of the center, forming an arc-like shape with a physical size of $50 \mathrm{AU}$. Second, the disk is surrounded by a tenuous and smooth envelope ${ }^{2}$ with a radius of $435 \mathrm{AU}$ with a PA of $167^{\circ}$. The envelope contains spiral-like patterns along the major axis. Adopting the dust temperature of $\sim 31 \mathrm{~K}$ measured by Hara et al. (2013), we estimate a disk plus envelope mass of $0.17 \pm 0.06 M_{\odot}$ (assuming a dust opacity $\kappa$ of $0.88 \pm 0.01 \mathrm{~cm}^{2} \mathrm{~g}^{-1}$ for a population dominated by grains with thin ice mantles, Ossenkopf \& Henning 1994). The minorto-major axis ratio indicates an inclination of $70^{\circ}$ with respect to the line of sight.

\footnotetext{
The Common Astronomy Software Applications tool by the ALMA staff as part of its Quality Assurance (QA) process.

2 We refer to this term as envelope in a more restrictive way than usual, since this is its inner flattened component.
}

\subsection{Molecular emission}

The CO (2-1) emission at velocities in the \pm 4 to $\pm 7 \mathrm{~km} \mathrm{~s}^{-1}$ range with respect to the systemic velocity of the source $\left(V_{\mathrm{lsr}} \sim\right.$ $3.6 \mathrm{~km} \mathrm{~s}^{-1}$ ) shows an elongated component that arises perpendicular to the envelope major axis and lies onto the plane-of-sky because of the large source inclination. This molecular component originates at a distance of $\sim 90-130$ AU from the disk center and the rotation axis, near the region of dust minimum outside the border of the disk (CO channel map in Fig. 2), where the dust spiral structures are connected to the disk. In the blueshifted side of the envelope, this component is extended along the northeast, whereas in the redshifted side it is extended in the opposite direction, along the southwest. At 90-130 AU, the escape velocity of the system is $4.8 \mathrm{~km} \mathrm{~s}^{-1}$ to $5.8 \mathrm{~km} \mathrm{~s}^{-1}$ with respect to the systemic velocity of the source (for our estimated stellar mass of $\left.1.70 \pm 0.04 M_{\odot}\right)$. This extended gas is therefore gravitationally unbound. The $\mathrm{CO}$ emission shows two features that are common in molecular outflows: it has an approximately symmetrical limb brightened conical cavity (lower left panel of Fig. 2), and there is evidence that the gas accelerates outwards. In both lobes, the cone vertex is offset from the rotation axis, showing that the outflow is launched far from the disk center. We therefore detect a molecular outflow that is launched (carrying out mass and angular momentum) outside the disk, far from the putative YSO. This type of outflow is different from the outflows observed so far, which are centered around the YSO, and it is not predicted in the classical picture of the local disk-outflow dynamics (Blandford \& Payne 1982; Shu et al. 1994; Pudritz et al. 2007). The highest $\mathrm{CO}$ velocities $\left(V_{\text {blue }}<-3.0 \mathrm{~km} \mathrm{~s}^{-1}\right.$ and $V_{\text {red }}>10 \mathrm{~km} \mathrm{~s}^{-1}$ ) are confined within $70 \mathrm{AU}$ of the inner disk. They suggest that BHB07-11 is a strongly perturbed disk (see Appedix A for a brief discussion).

The $\mathrm{C}^{18} \mathrm{O}(2-1)$ emission shows a clear velocity gradient that is fairly well aligned with the source long axis. This implies a rotation axis with a PA of $77^{\circ}$ in the plane of the sky. The position-velocity (PV) diagram pattern is consistent with Keplerian rotation (lower right panel of Fig. 2).

The $\mathrm{H}_{2} \mathrm{CO}\left(3_{0,3}-2_{0,2}\right)$ emission is confined to the dust envelope and also presents a velocity gradient with respect to the source center. At larger radii (120-435 AU), the spectrum profile of $\mathrm{H}_{2} \mathrm{CO}$ has a pronounced asymmetry with redshifted absorption (upper panel in Fig. 3) that is typical of infall gas motions in contracting clouds (e.g., Leung \& Brown 1977; Myers et al. 1996; Pineda et al. 2012; Evans et al. 2015b).

\section{Disk-envelope transition zone}

The transition zone between disk and envelope is crucial for understanding the disk evolution, especially for how the disk is assembled. The large-scale spiral structures are unlikely to originate in gravitational instabilities because the spirals are (1) extended structures outside the disk, and (2) not massive enough to be self-gravitating (see Appendix B). Instead, these spirals may be infall streams spiralling inward onto the disk because most of the infall motion is converted into the rotation motion. In fact, we show now that the gas kinematics at the outer spiral arm shows a sharp transition that matches the feature of a centrifugal barrier well.

The $\mathrm{H}_{2} \mathrm{CO} \mathrm{PV}$ cut along the major axis shows a clear discontinuity between the envelope and the inner disk kinematics (lower right panel in Fig. 3). The molecular emission exceeds the Keplerian curves in velocity and reaches a maximum at $225 \mathrm{AU}\left(1^{\prime \prime} .55\right)$ at the northern side and $121 \mathrm{AU}(0, .83)$ at 

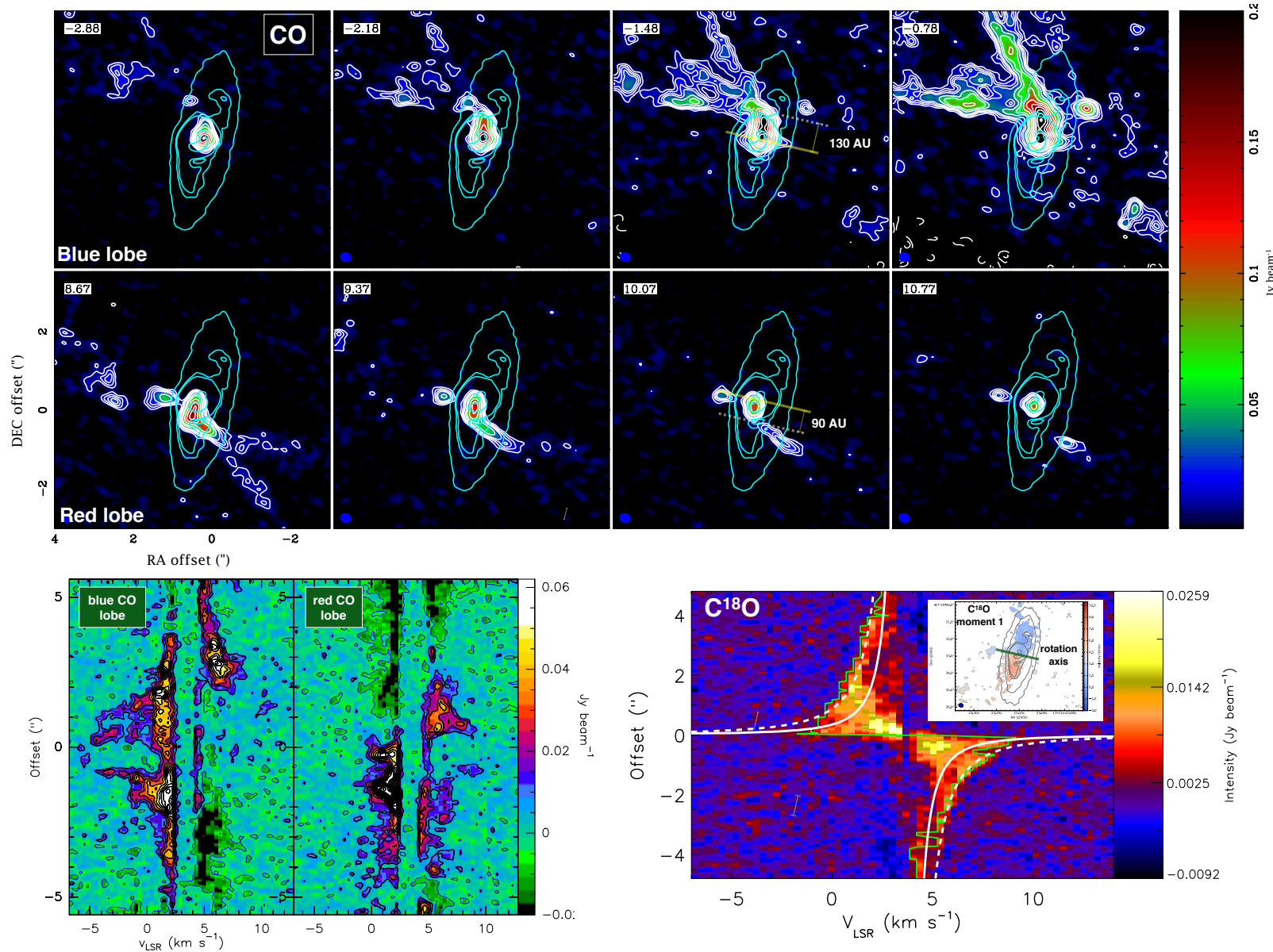


(cyan contours with the same intensity levels as Fig. 1). The velocity channels are labeled in the upper left corner of each panel. The channel spacing is binned to $0.7 \mathrm{~km} \mathrm{~s}^{-1}$. The yellow arrow line in channels $-1.48 \mathrm{~km} \mathrm{~s}^{-1}$ and $10.07 \mathrm{~km} \mathrm{~s}^{-1}$ indicates the rotation axis of the disk. Lower left panel: PV plots taken from cuts transversal to the outflow axis in each lobe. The cavity pattern is indicated by the large opening angle. Lower right panel: $\mathrm{C}^{18} \mathrm{O} \mathrm{PV}$ diagram from a cut along the source long axis. The dashed line shows the best Keplerian fit to the $3 \sigma$ envelope (green line), following the method proposed by Seifried et al. (2016). The fit corresponds to a dynamical mass of $1.70 \pm 0.04 M_{\odot}$, while the white line shows the fit of Hara et al. (2013) for a mass of $0.73 M_{\odot}$. The inset shows the $\mathrm{C}^{18} \mathrm{O}$ velocity field for a pixel intensity brighter than $50 \%$ of the peak flux $\left(25 \mathrm{mJy}\right.$ beam $\left.^{-1}\right)$. The contours refer to the continuum emission, and the green arrow shows the rotation axis. The synthesized beam is 0 '! $20 \times 0$ '? 24 for the continuum, 0 '!20 $200^{\prime \prime} .25$ for the $\mathrm{CO}$ data, and $0^{\prime \prime} .21 \times 0^{\prime \prime} .27$ for the $\mathrm{C}^{18} \mathrm{O}$ data.

the southern side. It is then significantly decelerated between a radius of $\sim 110-188$ AU $(0$, ! $76-1$ '!30) at the northern side and $\sim 87-107$ AU $\left(00^{\prime \prime} 6-00^{\prime \prime} \cdot 74\right)$ at the southern side. This velocity feature matches the centrifugal barrier in existing observational (e.g., Sakai et al. 2014; Oya et al. 2016) and (magneto)hydrodynamics models well (Zhao et al. 2016). The velocity peak is produced when the infalling gas piles up at their centrifugal radii. The flow dynamics across this centrifugal barrier is mainly dominated by rotation, while the infall motion decreases drastically. Compared with the dust continuum map in Fig. 1, the location of the centrifugal barrier is at the tip of the spiral structures, with the northern lobe slightly farther out than the southern lobe.

The gas appears to flow gradually along the spiral arms onto the disk, where infall and rotation velocities are reduced, as indicated by the cavity feature in the PV diagram (lower right panel in Fig. 3). However, in the inner $\pm 0^{\prime \prime}$.6 region, which represents the disk, the kinematics is mainly dominated by the central protostar, and the gas resumes its Keplerian speed.

It is clear that on both sides of the disk, the launching base of the main outflow coincides with the intersection of the disk and spiral, where the infall flow lands on the disk (Fig. 2). This location is the most probable site satisfying the conditions of magneto-centrifgual mechanism (Blandford \& Payne 1982), because the infall drags the magnetic field lines inward and creates large pinching angles for the field lines (Zhao et al. 2016). In comparison, field lines inside the Keplerian disk are much less pinched because of the rotation support and the lack of fast infall motions. Therefore, more sophisticated non-ideal magnetohydrodynamics (MHD) mechanisms are required for launching wind on the disk itself, which is likely to be weak and unsteady (e.g., Bai \& Stone 2017; Zhu \& Stone 2017).

Our work thus shows that the most prominent magnetocentrifugal outflows should be ejected near the landing site of the gas infall onto the disk, while less notable outflow components are launched in the inner radius of the disk (CO component aligned with the rotation axis in the redshifted channels of Fig. 2). In this sense, the outflow observed by Bjerkeli et al. (2016) is likely to belong to the weaker disk wind component; however, if the landing site of the gas infall in their case is not on the disk edge along the equator, but rather on the upper disk surface, it may still fit the physical pictures we presented here. It is worth pointing out that in both observations, the outflows are not symmetric, as in the classical disk wind picture. The landing 

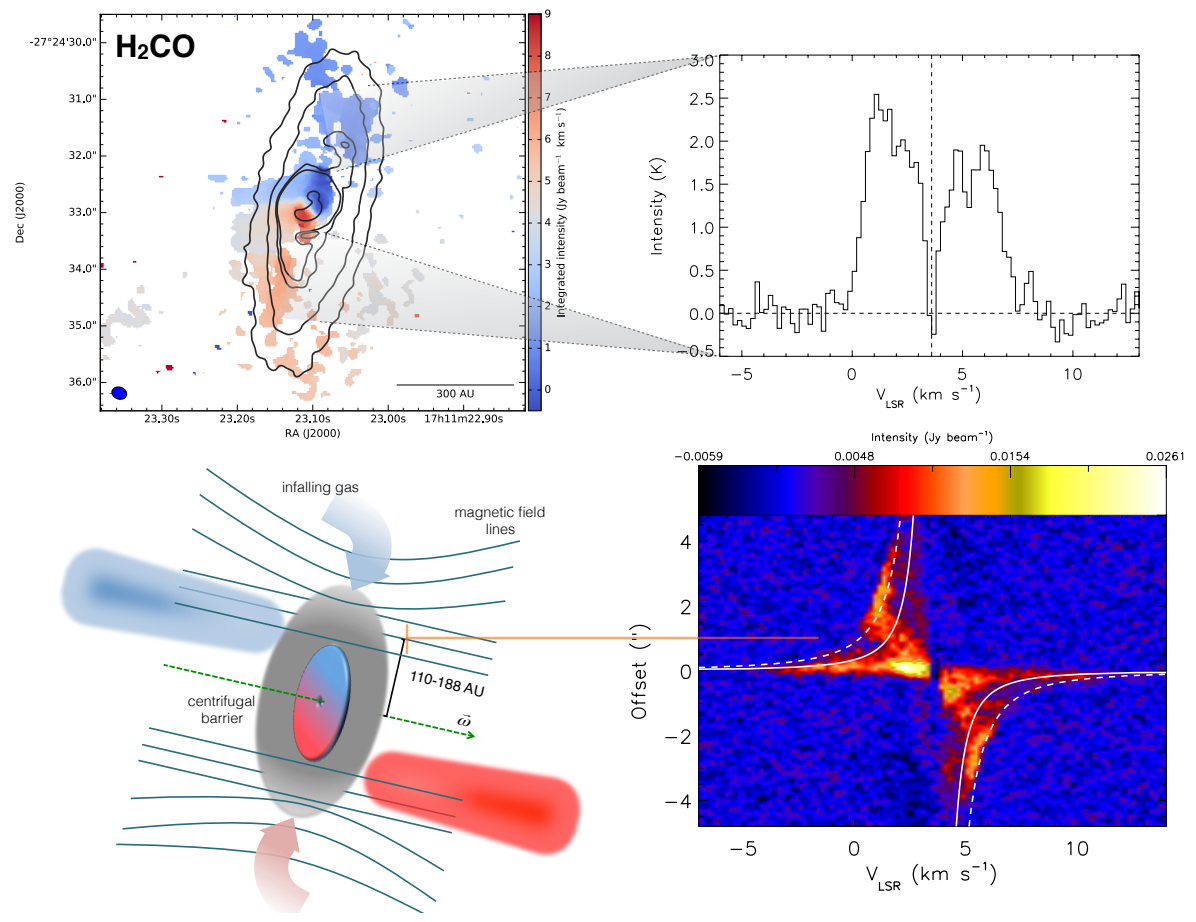

Fig. 3. Upper panel: velocity field of the $\mathrm{H}_{2} \mathrm{CO}$ emission with respect to the continuum emission (contours). The inset shows an asymmetric line profile taken from a radius between 120 and $435 \mathrm{AU}$ where the blueshifted emission is brighter than the redshifted one, which is typical of infall motions. The thick vertical dashed line shows the ambient velocity according to previous observations $\left(V_{\mathrm{lsr}} \sim 3.6 \mathrm{~km} \mathrm{~s}^{-1}\right.$, Onishi et al. 1999; Hara et al. 2013). The horizontal dashed line shows the continuum-removed zero-level baseline. Lower panels: scheme showing outflows powered at the disk edge that are due to the magnetic field enhancement. The right panel shows the PV diagram of the $\mathrm{H}_{2} \mathrm{CO}$ emission with the same Keplerian curves as in Fig. 2. The discontinuity in the velocity distribution pattern around $\sim 1^{\prime \prime}$.0 indicates the change in kinematics probably caused by the centrifugal barrier. The discontinuity is preceded by a velocity peak (better seen in the northern lobe as a "knee" in the PV plot) and followed by a sharp increase in radial velocity in the inner $0.6^{\prime \prime}$ as a result of the disk rotation. site of the infall streams onto the disk is indeed typically asymmetric; hence, symmetric bipolar outflows (not jets) should be rather rare in this framework.

\section{Conclusions}

This paper shows that the high-sensitivity image fidelity and angular resolution of ALMA reveals new phenomena associated with the formation of stars, and it shows that the dynamics of the disk-outflow systems around YSOs is far more complex than initially thought. In particular, outflows can be launched at a distance of $\sim 90-130 \mathrm{AU}$ from the rotational axis, where gas infall along the extend spiral structures lands on the disk. This scenario is consistent with the standard magneto-centrifugal mechanism that requires a large pinching angle of the magnetic field lines, which is most likely to be achieved at these infall landing sites. Furthermore, the high-resolution data allow us to see the detailed morphologies in the transition zone of the disk and envelope, and to identify the location of the centrifugal barrier as on the outer spiral arm.

Acknowledgements. This paper makes use of the following ALMA data: DS/JAO.ALMA\#2013.1.00291.S. ALMA is a partnership of ESO (representing its member states), NSF (USA) and NINS (Japan), together with NRC (Canada), NSC and ASIAA (Taiwan), and KASI (Republic of Korea), in cooperation with the Republic of Chile. The Joint ALMA Observatory is operated by ESO, AUI/NRAO and NAOJ. G.A.P.F. acknowledges the partial support from CNPq and FAPEMIG (Brazil). J.M.G. acknowledges support from MICINN AYA2014-57369-C3-P and the MECD PRX15/00435 grants (Spain). P.C., B.Z. and M.E. acknowledge support of the European Research Council (ERC; project PALs 320620). WV acknowledges support from the ERC through consolidator grant 614264 . The data reported in this paper are archived in the ALMA Science Archive.

\section{References}

Alves, F. O., \& Franco, G. A. P. 2007, A\&A, 470, 597

Alves, F. O., Franco, G. A. P., \& Girart, J. M. 2008, A\&A, 486, L13
Alves, J., Lombardi, M., \& Lada, C. J. 2007, A\&A, 462, L17

Armitage, P. J. 2011, ARA\&A, 49, 195

Bai, X.-N., \& Stone, J. M. 2017, ApJ, 836, 46

Bjerkeli, P., van der Wiel, M. H. D., Harsono, D., Ramsey, J. P., \& Jørgensen, J. K. 2016, Nature, 540, 406

Blandford, R. D., \& Payne, D. G. 1982, MNRAS, 199, 883

Bodenheimer, P. 1995, ARA\&A, 33, 199

Brinch, C., \& Hogerheijde, M. R. 2010, A\&A, 523, A25

Brooke, T. Y., Huard, T. L., Bourke, T. L., et al. 2007, ApJ, 655, 364

Cotten, T. H., \& Song, I. 2016, ApJS, 225, 15

Covey, K. R., Lada, C. J., Román-Zúñiga, C., et al. 2010, ApJ, 722, 971

Duarte-Cabral, A., Chrysostomou, A., Peretto, N., et al. 2012, A\&A, 543, A140 Evans, M. G., Ilee, J. D., Boley, A. C., et al. 2015a, MNRAS, 453, 1147

Evans, II, N. J., Di Francesco, J., Lee, J.-E., et al. 2015b, ApJ, 814, 22

Forbrich, J., Lada, C. J., Muench, A. A., Alves, J., \& Lombardi, M. 2009, ApJ, 704, 292

Franco, G. A. P., Alves, F. O., \& Girart, J. M. 2010, ApJ, 723, 146

Frau, P., Girart, J. M., Beltrán, M. T., et al. 2010, ApJ, 723, 1665

Frau, P., Girart, J. M., Beltrán, M. T., et al. 2012, ApJ, 759, 3

Hara, C., Shimajiri, Y., Tsukagoshi, T., et al. 2013, ApJ, 771, 128

Ilee, J. D., Boley, A. C., Caselli, P., et al. 2011, MNRAS, 417, 2950

Konigl, A., \& Pudritz, R. E. 2000, Protostars and Planets IV (University of Arizona Press), 759

Leung, C. M., \& Brown, R. L. 1977, ApJ, 214, L73

Lombardi, M., Alves, J., \& Lada, C. J. 2006, A\&A, 454, 781

Myers, P. C., Mardones, D., Tafalla, M., Williams, J. P., \& Wilner, D. J. 1996, ApJ, 465, L133

Onishi, T., Kawamura, A., Abe, R., et al. 1999, PASJ, 51, 871

Ossenkopf, V., \& Henning, T. 1994, A\&A, 291, 943

Oya, Y., Sakai, N., López-Sepulcre, A., et al. 2016, ApJ, 824, 88

Pineda, J. E., Maury, A. J., Fuller, G. A., et al. 2012, A\&A, 544, L7

Pudritz, R. E., Ouyed, R., Fendt, C., \& Brandenburg, A. 2007, Protostars and Planets V, 277

Riaz, B., Martín, E. L., Bouy, H., \& Tata, R. 2009, ApJ, 700, 1541

Román-Zúñiga, C. G., Lada, C. J., Muench, A., \& Alves, J. F. 2007, ApJ, 664, 357

Sakai, N., Sakai, T., Hirota, T., et al. 2014, Nature, 507, 78

Seifried, D., Sánchez-Monge, Á., Walch, S., \& Banerjee, R. 2016, MNRAS, 459, 1892

Shu, F., Najita, J., Ostriker, E., et al. 1994, ApJ, 429, 781

Shu, F. H., Najita, J. R., Shang, H., \& Li, Z.-Y. 2000, Protostars and Planets IV, 789

Toomre, A. 1964, ApJ, 139, 1217

Zhao, B., Caselli, P., Li, Z.-Y., et al. 2016, MNRAS, 460, 2050

Zhu, Z., \& Stone, J. M. 2017, ArXiv e-prints [arXiv: 1701. 04627]

Zinnecker, H., McCaughrean, M. J., \& Rayner, J. T. 1998, Nature, 394, 862 


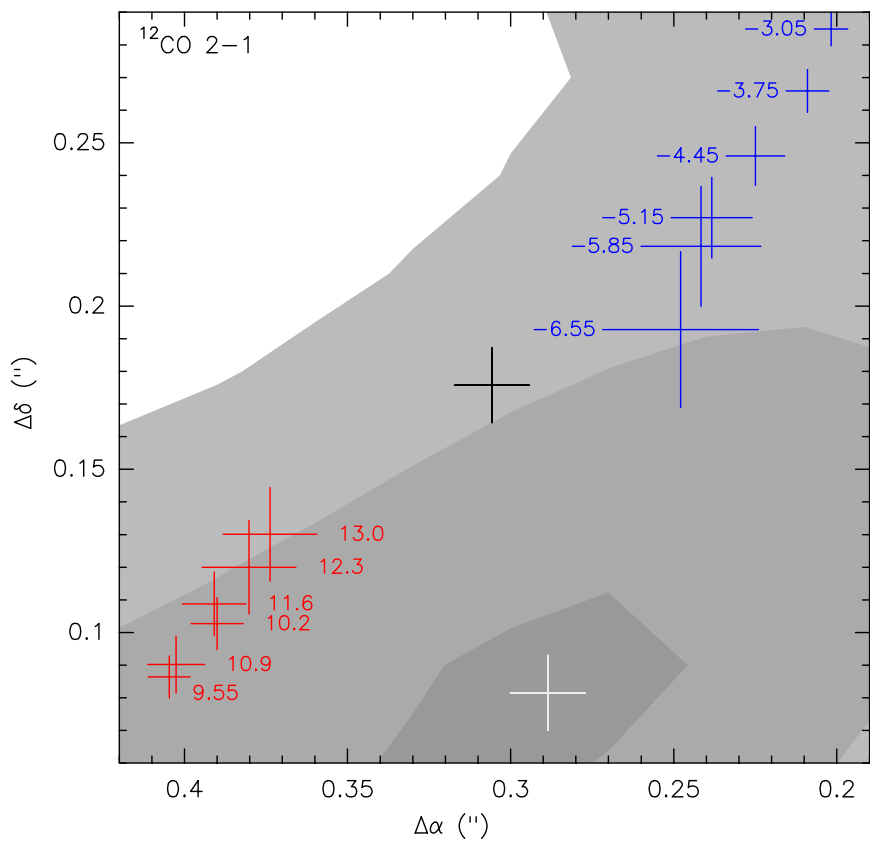

Fig. A.1. Zoomed-in view of the disk center. The gray scale is the dust continuum emission. The color crosses are the peak of $\mathrm{CO}$ emission at high-velocity channels. The length of the cross indicates the uncertainty of the peak position. The numbers next to the crosses indicate the corresponding velocity channel (in $\mathrm{km} \mathrm{s}^{-1}$ ). The black cross is the averaged center position for the high-velocity channels, and the white cross shows the dust emission peak.

\section{Appendix A: High CO velocity components}

The highest velocity channels of the $\mathrm{CO}$ emission $\left(V_{\mathrm{lsr}}<\right.$ $-3 \mathrm{~km} \mathrm{~s}^{-1}$ for the blueshifted gas and $>10 \mathrm{~km} \mathrm{~s}^{-1}$ for redshifted gas) are confined to the disk. We performed Gaussian fits to compute the peak position of these components and found that they appear to be located approximately in the same direction (Fig. A.1). As the velocity increases, the blue and red peak positions move closer to each other in an approximately linear manner, with the averaged position indicating the gravitational center. The center of this line indicates that the kinematical center is 14 AU removed from the dust peak.

This offset between the gravitational center and the dust peak indicates that BHB07-11 possibly hosts a multiple system. The disk asymmetry could be a barely resolved binary system, since BHB07-11 is a young source (0.1-0.2 Myr, Brooke et al. 2007) and its disk does not seem to be massive enough to develop gravitational instabilities (Appendix B).

\section{Appendix B: Modeling gravitational instabilities}

Circumstellar disks with masses comparable to their central protostar can develop gravitational instabilities that are due to their self-gravity (Toomre 1964). This effect produces spiral density waves that regulate the mass accretion process. In order to verify whether the azimuthal asymmetries seen in the disk are due to unresolved spirals, we used a model representing an early Class I object with size and mass very similar to the disk in BHB0711 . The model was a self-gravitating disk of $0.17 M_{\odot}$ surrounding a solar-type star (Evans et al. 2015a). The model spanned $130 \mathrm{AU}$ and was placed at the same distance $(145 \mathrm{pc})$ and inclination $\left(70^{\circ}\right)$ as BHB07-11. The peak temperature and density of the model were $330 \mathrm{~K}$ and $5.7 \times 10^{13} \mathrm{~cm}^{-3}$, respectively, and
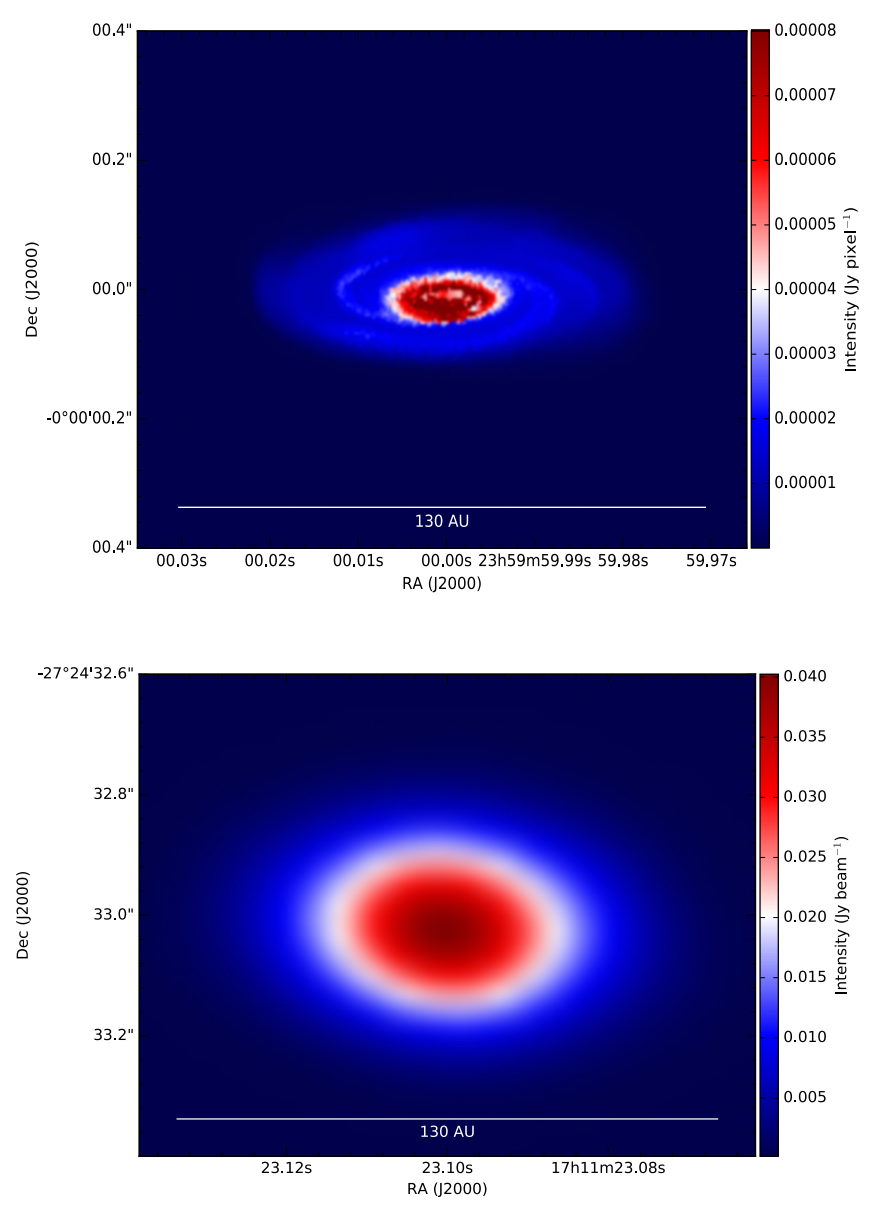

Fig. B.1. Upper panel: self-gravitating disk model (Evans et al. 2015a). The spirals produced by the gravitational instabilities are visible at radii larger than $20 \mathrm{AU}$. Lower panel: simulation of this model using the same antenna configuration as our observations. The angular resolution of the simulated map is similar to our data $\left(0^{\prime \prime} .25 \times 0,20\right)$.

were confined within a radius of $10 \mathrm{AU}$ in the disk. At larger radii, the temperature dropped below $50 \mathrm{~K}$. Through non-local thermodynamic equilibrium radiative transfer processing, using LIME (line modeling engine - Brinch \& Hogerheijde 2010), we observed that the optical depth varied across the disk structure; it was optically thinner in the inner disk. Given the similarities between the physical properties of the model and BHB07-11, we used CASA to simulate an observation of this model under the same conditions as our observations; that is, we simulated band 6 observations of the model with the same continuum bandwidth as our spectral setup $(2.3 \mathrm{GHz})$ and the same antenna configuration. We also added thermal noise using the atmospheric profiles of the ALMA site, including a realistic precipitable water vapor level.

As shown in the lower panel of Fig. B.1, the simulations are not able to reproduce the spiral structures because they are too small to be resolved in our observations. This implies that a larger and more massive disk than that of BHB07-11 is necessary to develop gravitational instabilities that are detectable at $145 \mathrm{pc}$ distance. Even more prominent spirals produced by a more massive disk model $\left(0.39 M_{\odot}\right.$, Ilee et al. 2011) were not detected in our simulations. Therefore, the azimuthal asymmetries must be produced by another mechanism. 